\title{
Photosensitive Ionomer. II. Preparation of Mercurous Copoly(vinyl alcohol-acrylate) and Properties of the Plate
}

\author{
Kazuyuki Sugita and Shin Suzuki \\ Department of Graphic Engineering, Faculty of Engineering, \\ Chiba University, 1-33, Yayoi-cho, Chiba, Japan.
}

(Received December 25, 1970)

\begin{abstract}
The disproportionating reaction of mercurous acetate was used in conjunction with polymeric carboxylic acid in order to produce a new type of photosensitive system. As for the polymeric acid, various copolymers of vinyl alcohol and acrylic acid were prepared by radical copolymerization of vinyl acetate and acrylic acid with varying monomer ratios and volumes of xylene used as a solvent. The polymeric acid was neutralized with sodium hydroxide and then converted to a mercurous salt. Photosensitive plates were prepared by coating glass plates with a dispersion of mercurous copoly(vinyl alcohol-acrylate).

It was found that the plates were sensitive to ultraviolet light, and that a dark brown image was formed by a printing-out process. The latent image was proved to be developed by hydroquinone. Photographic image formation was due to metallic mercury which was isolated as a product of the disproportionating reaction. When immersed in an acetic acid solution, the photosensitive layer of the unexposed part swelled and became detached from the plate, but the layer of the exposed part was unchanged, and a relief image was fixed on the plate. The image formation was ascribed to ionic crosslinking by mercuric ions formed in the disproportionating reaction of mercurous ion.
\end{abstract}

KEY WORDS Photosensitive Ionomer / Mercurous Copoly(vinyl alcohol-acrylate)/ Disproportionation / Photographic Image/ Latent Image / Development / Metallic Mercury/Relief Image/Ionic Crosslinking / Mercuric Ion /

Ionomer ${ }^{1}$ has been developed since 1964 for the purpose of improving mechanical properties (e.g., hardness, tensile strength, wear resisting and so on) of thermo-plastic polymers. The ionomer has an ionically crosslinked network structure. In most cases, the ionic crosslinkages are $-\mathrm{COO}^{-} \ldots \mathrm{M}^{+} \ldots \mathrm{O}^{-} \mathrm{CO}-$ or $-\mathrm{COO}^{-} \ldots$ $\mathrm{M}^{++} \ldots \mathrm{O}^{-} \mathrm{CO}$ - formed by adding mono- or divalent metallic ions, respectively, to a linear polymer with side chains of carboxyl groups. As the strength of the ionic crosslinking depends on the valence state of the metallic ion, the ionomer which is crosslinked by a metallic ion of variable valence on exposure may become a new photosensitive polymer.

On the other hand, it is well known that mercurous acetate disproportionates to mercuric acetate and metallic mercury on exposure to light, ${ }^{2}$ as illustrated in eq 1.

$$
2 \mathrm{CH}_{3} \mathrm{COOHg} \longrightarrow\left(\mathrm{CH}_{3} \mathrm{COO}\right)_{2} \mathrm{Hg}+\mathrm{Hg}
$$

Photoreduction of the mercurous ion into metallic mercury is similar to that of the silver ion, and the mercury is available for photographic image formation. Moreover, the mercuric ion which is produced by photooxidation of mercurous ion will form crosslinkages, if some linear polymer has side chains of mercurous carboxylate groups

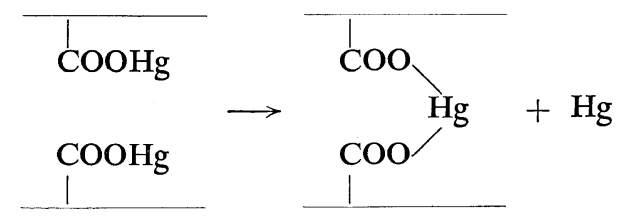

(see eq 2). Therefore, a polymeric mercurous compound is expected to be a new photosensi- 
tive polymer which hardens by ionic crosslinking, and may be used for lithographic image formation.

In the previous paper, ${ }^{3}$ the authors reported on the photosensitivity of an emulsion composed of mercurous acrylate and poly(vinyl alcohol) which can be regarded as a model emulsion of linear polymer with side chains of mercurous carboxylate and hydroxyl groups. The following conclusions were formed.

(1) Mercurous acrylate was sensitive to light ranging from 250 to $340 \mathrm{~nm}$, and formed a dark brown image with density increase of about 0.6 by a printing-out process.

(2) The latent image could be brought out with a reducing developer such as hydroquinone or metol.

(3) Metallic mercury, isolated by photoreduction of the mercurous ion, contributed to photographic image formation.

(4) Though the photographic image on the plate was not broken by fogging or fading during storage for several months, fixation was unsuccessful in this case, for no solvents which preferentially dissolved mercurous acrylate not disproportionated were found.

In this paper, mercurous copoly(vinyl alcohol-acrylate) was prepared as an example of a polymeric mercurous compound and a plate coated with the copolymer was studied for supplying a new photosensitive polymer with both developing and fixing ability. Moreover, the strength of the ionic crosslinkages of $-\mathrm{COO}^{-}$ $\ldots \mathrm{Hg}^{+} \ldots \mathrm{Hg}^{+} \ldots \mathrm{O}^{-} \mathrm{CO}-$ and $-\mathrm{COO}^{-} \ldots \mathrm{Hg}^{++}$ ... $\mathrm{O}^{-} \mathrm{CO}-$ was deduced.

\section{EXPERIMENTAL}

\section{Materials}

Acrylic acid, vinyl acetate, and xylene were purified by distillation of commercially available reagents. The other reagents used were chemicals of extra pure grade.

\section{Apparatus}

Titration was performed by using a Yokokawa $\mathrm{pH}$ Meter KPH-51B. The wedge spectrogram was obtained with a Ushio Xenon Arc Lamp UXL-500D and a Narumi Monochromator RM23. Photosensitive plates were irradiated by a Ushio Low-pressure Mercury Lamp ULI-309 (a chemical lamp) from a distance of $15 \mathrm{~cm}$.

\section{Preparation of Copoly(Vinyl Alcohol-Acrylic Acid)}

This copolymer was prepared by referring to the method used to obtain poly(vinyl alcohol) ${ }^{4}$. Vinyl acetate, acrylic acid, benzoyl peroxide, and xylene of the quantity indicated in Table I were placed in a polymer tube (diameter, $2.5 \mathrm{~cm}$; length, $11 \mathrm{~cm}$ ). After evacuating the air, the tube was sealed off under reduced pressure in nitrogen. The tube was heated in a water bath kept at $80^{\circ} \mathrm{C}$ and polymerization was allowed to proceed at this temperature for $3 \mathrm{hr}$. The polymerization products were dissolved in $80 \mathrm{ml}$ of boiling methanol in a $200-\mathrm{m} l$ flask equipped with a condenser. Five per cent sodium

Table I. Raw materials ${ }^{a}$ and characterization of copoly(vinyl alcohol-acrylic acid)

\begin{tabular}{ccccccc}
\hline $\begin{array}{c}\text { Copolymer } \\
\text { number }\end{array}$ & $\begin{array}{c}\text { Acrylic acid, } \\
\mathrm{g}\end{array}$ & $\begin{array}{c}\text { Vinyl acetate, } \\
\mathrm{g}\end{array}$ & Xylene, $\mathrm{g}$ & Yield, wt\% & $\begin{array}{c}\text { Acrylic acid in } \\
\text { copolymer, wt } \%\end{array}$ & $\eta_{\mathrm{sp}} / C$ \\
\hline 201 & 0.2 & 9.8 & 1.0 & 92.3 & 0.14 & 2.40 \\
202 & 0.2 & 9.8 & 2.0 & 98.1 & 0.15 & 1.72 \\
205 & 0.2 & 9.8 & 5.0 & 69.1 & 0.21 & 1.56 \\
210 & 0.2 & 9.8 & 10.0 & 44.7 & 0.25 & 1.20 \\
501 & 0.5 & 9.5 & 1.0 & 25.3 & 2.4 & 69.0 \\
502 & 0.5 & 9.5 & 2.0 & 18.4 & 3.8 & 41.4 \\
505 & 0.5 & 9.5 & 5.0 & 16.9 & 4.3 & 10.5 \\
510 & 0.5 & 9.5 & 10.0 & 19.3 & 6.8 & 7.0 \\
1002 & 1.0 & 9.0 & 2.0 & 38.5 & 17.4 & 35.2 \\
1005 & 1.0 & 9.0 & 5.0 & 35.2 & 17.8 & 12.8 \\
1010 & 1.0 & 9.0 & 10.0 & 34.2 & 23.7 & 6.7 \\
\hline
\end{tabular}

\footnotetext{
a $0.1 \mathrm{~g}$ of benzoyl peroxide was used as an initiator in each case.
} 
methoxide in methanol was added to the refluxing polymer solution in $2-\mathrm{m} l$ portions at intervals of $5 \mathrm{~min}$. Ten $\mathrm{m} l$ of the solution was sufficient to catalyze the methanolysis. After addition of sodium methoxide solution, refluxing was continued for a further $15 \mathrm{~min}$ and the flask was left to cool. Eighty $\mathrm{m} l$ of ethyl ether was added to the flask for complete precipitation of the polymer. The precipitates were separated by decantation and centrifugation at $5000 \mathrm{rpm}$, and washed several times with a mixed solvent of methanol and ethyl ether (1:1 by volume) and then dried.

The molar ratio of acrylic acid incorporated in the copolymer was determined by titrating carboxyl groups with $0.1-N$ sodium hydroxide. Molecular weight was estimated relatively by measuring a reduced viscosity, $\eta_{\mathrm{sp}} / C$, at $30^{\circ} \mathrm{C}$ on an aqueous solution of the copolymer $(0.25 \mathrm{~g} /$ $100 \mathrm{~m} l$ ). As the correlation curve between $\eta_{\mathrm{sp}} / C$ and $C$ was not a straight line, intrinsic viscosity

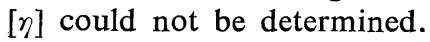

Preparation of Mercurous Copoly(Vinyl AlcoholAcrylate) and Photosensitive Plates

One $\mathrm{g}$ of copoly(vinyl alcohol-acrylic acid) was dissolved in $50 \mathrm{ml}$ of water and exactly neutralized by $1-N$ sodium hydroxide using a $\mathrm{pH}$ meter. Mercurous nitrate monohydrate was dissolved in $c a .6 \%$ nitric acid $(14 \mathrm{~g} / 80 \mathrm{ml})$, and concentrations of mercurous ion and nitrate ion were determined by titrating with $0.1-N$ potassium hydroxide $\left[\mathrm{Hg}^{+}\right]=0.49 \mathrm{~N}$, and $\left[\mathrm{NO}_{3}^{-}\right]=1.12 \mathrm{~N}$. Under vigorous agitation in the dark, the mercurous nitrate solution was added to the sodium copoly(vinyl alcohol-acrylate) solution, and then the solution turned a milky white emulsion. In this treatment the volume of the nitrate solution was controlled so that the equimolar amount of nitrate ion to sodium ion in the copolymer solution was sustained. The mercurous salt of the copolymer was separated by centrifugation at $10000 \mathrm{rpm}$. The quantity of mercurous ions in the mother liquid was too small to be titrated. Mercurous ions in the nitrate solution were thus regarded as being mainly quantitatively fixed to side chains of the copolymer. The mercurous acrylate component in mercurous copoly(vinyl alcohol-acrylate) was calculated and is shown in Table II. The mercu- rous salt precipitated was again uniformly dispersed in a small quantity of water, spread over glass plates, and left to dry.

\section{Analysis of Metallic Mercury and Mercuric Ion}

The photosensitive layer was scraped off the plate $(96 \times 96 \mathrm{~mm})$ after exposure for $50 \mathrm{~min}$, and the mercurous copoly(vinyl alcohol-acrylate) $(50-70 \mathrm{mg}$ ) was refluxed in $10 \mathrm{ml}$ of dilute sulfuric acid $(1: 20)$ for $2 \mathrm{hr}$. Small drops of metallic mercury, which were obtained by this treatment, together with the white residue of the copolymer, were washed with $10 \mathrm{~m} l$ of water and weighed. The sulfuric acid solution was combined with the wash liquid. To the combined solution, $1.28 \mathrm{~m} l$ of $0.5 \mathrm{~N}$ hydrochloric acid was added to precipitate mercurous chloride. The white precipitates were filtered off and washed with $10 \mathrm{~m} l$ of $0.03-N$ hydrochloric acid. The filtrate was neutralized to $\mathrm{pH} 10.0$ by adding $10-15 \mathrm{~m} l$ of $1-N$ sodium hydroxide, to give a reddish brown precipitate of mercuric oxide. These precipitates were washed with water, dissolved in $10 \mathrm{ml}$ of nitric acid $(1: 10)$ and submitted to EDTA titration, using xylenol orange as a metal indicator.

\section{RESULTS}

\section{Photosensitive Layer on the Plate}

The layers made from Copolymer 202, 205, 210, 505, and 510 (see Table I) were smooth and transparent, but those from Copolymer 201, 501, and 502 were partly white and turbid. The layers from Copolymer 1002, 1005, and 1010 were cracked or powdery, and could not be used for photosensitive plates. The wedge spectrogram showed that the layer colored dark brown on exposure to light ranging from 250 to $325 \mathrm{~nm}$. This result is similar to that of mercurous acrylate reported in the previous paper. Irradiation was then carried out by using a low-pressure mercury lamp.

\section{Development}

Each photosensitive plate was divided into four parts. The first part was irradiated for $50 \mathrm{~min}$, the second for $2 \mathrm{~min}$, the third for $1 \mathrm{~min}$ and the last was not irradiated at all. The first turned dark brown, but the second and the third were still transparent and could not be dis- 


\section{K. Sugita and S. SuzukI}

Table II. Properties of photosensitive layer

\begin{tabular}{cccccc}
\hline $\begin{array}{c}\text { Copolymer } \\
\text { number }\end{array}$ & $\begin{array}{c}\text { Hg acrylate in } \\
\text { copolymer }^{\mathrm{a}}\end{array}$ & Film formation & Photosensitivity & Development & Fixation \\
\hline 201 & 0.23 & $\triangle$ & $\bigcirc$ & $\triangle$ & $\times$ \\
202 & 0.25 & $\bigcirc$ & $\bigcirc$ & $\triangle$ & $\times$ \\
205 & 0.35 & $\bigcirc$ & $\bigcirc$ & $\triangle$ & $\times$ \\
210 & 0.41 & $\bigcirc$ & $\bigcirc$ & $\triangle$ & $\times$ \\
501 & 3.86 & $\triangle$ & $\bigcirc$ & $\bigcirc$ & $\bigcirc$ \\
502 & 6.00 & $\triangle$ & $\bigcirc$ & $\bigcirc$ & $\bigcirc$ \\
505 & 6.76 & $\bigcirc$ & $\bigcirc$ & $\bigcirc$ & $\triangle$ \\
510 & 10.04 & $\bigcirc$ & & & \\
1002 & 23.7 & $\times$ & & & \\
1005 & 24.2 & $\times$ & & & \\
1010 & 30.4 & $\times$ & & & \\
\hline
\end{tabular}

a Calculated values (in wt $\%$ ) by assuming that the quantity of mercurous ions in the nitrate solution was quantitatively fixed to side chains of each copolymer.

tinguished from the last. The plates made from Copolymers 501, 502, 505, and 510 were immersed in an aqueous alcoholic solution of hydroquinone $(1 \mathrm{~g}$ in $20 \mathrm{~m} l$ of water and $80 \mathrm{~m} l$ of alcohol). In a few minutes the second and the third parts turned dark grey, while the last did not. Development was completed in 10-15 min, and fogging was observed on the last part once development exceded $20 \mathrm{~min}$. In the case of the plates made from Copolymers 201, 202, 205, and 210 , the density increase on development was smaller.

\section{Fixation}

One half of each plate was irradiated for $50 \mathrm{~min}$, while the other was not. The plates made from Copolymers 501, 502, and 505 were placed in a mixed solvent of acetic acid $(15 \mathrm{ml})$, water $(15 \mathrm{ml})$, and alcohol $(70 \mathrm{ml})$. In $10-15 \mathrm{~min}$ the photosensitive layer of the unexposed part swelled and became detached from the plate, but that of the exposed part was unchanged. In the case of the plates from Copolymer 510, not only the layer of the unexposed part, but that of the exposed part, was slightly swollen and the image distorted. The unexposed layers from Copolymers 201, 202, 205 or 210 were never swollen and the image could not be fixed by this procedure.

The properties of the photosensitive layers are summarized in Table II.

Detection of Metallic Mercury and Mercuric Ions

The photosensitive plate made from Copolymer
505 was irradiated for $50 \mathrm{~min}$ and analyzed by the method described previously. $1.2 \mathrm{mg}$ of metallic mercury and $4.5 \times 10^{-6}$ molecules of mercuric ions ( 4 and $3 \mathrm{~mol} \%$, respectively, vs. mercurous ion before irradiation) were detected. In order to evaluate mercurous ions separated in the form of white precipitates of mercurous chloride, substitution titration was tried by $\mathrm{Mg}$-EDTA, but the end point of the titration was obscure and no significant value was obtained. Though the plates before irradiation, and after a period of $5 \mathrm{~min}$ irradiation, were analyzed in the same way, neither metallic mercury nor mercuric ions could be detected.

\section{DISCUSSION}

\section{Method of Preparing Photosensitive Plate}

The layers made from the copolymers, which were prepared by copolymerizing $1.0 \mathrm{~g}$ of acrylic acid with $9.0 \mathrm{~g}$ of vinyl acetate, were cracked or powdery. This may mean that too much mercurous acrylate decreases the film-forming ability of the copolymer. In the case of plates made by copolymerizing $0.2 \mathrm{~g}$ of acrylic acid with $9.8 \mathrm{~g}$ of vinyl acetate, immersion in a mixed solvent of acetic acid, water, and alcohol could not fix the image. When the amount of mercurous acrylate component is small, the copolymer does not show any swelling characteristics in contact with acetic acid. The copolymer must therefore contain a definite range of acrylic acid components in order to achieve 
optimum photosensitivity.

The influence of molecular weight on the properties of the photosensitive plates, which were made from the copolymers prepared by copolymerizing $0.5 \mathrm{~g}$ of acrylic acid with $9.5 \mathrm{~g}$ of vingl acetate was considered. The copolymer prepared by using $10.0 \mathrm{~g}$ of xylene could be regarded as having the smallest molecular weight of the four investigated. The image on the plate made therefrom was slightly swollen and distorted. The copolymer prepared by using $1.0-2.0 \mathrm{~g}$ of xylene was considered to be of comparatively large molecular weight. The photosensitive layer was partly white and turbid. 'This may have been due to the existence of an intermolecular force too strong to allow mercurous carboxylate groups to disperse homogeneously in polymer segments with hydroxyl groups. Molecular weight of the copolymer must therefore be moderate.

An aqueous solution of poly(vinyl alcohol) with a molecular weight of 22000 showed a reduced viscosity of 0.57 measured under the same conditions. Comparing it with the viscosity of the copolymers (see Table I), the authors assume that strong interactions exist between the polymer chains.

\section{Process of Image Formation}

The analytical results were not completely quantitative, because the white residue of copolymer seemed to contain metallic mercury and mercuric ions besides mercurous ions. However it was at least clarified that the mercurous ion disproportionates into metallic mercury and mercuric ions on exposure.

In addition to the experimental results reported in the previous paper, i.e., developing by the reducing agent and bleaching by the oxidizing agent, detection of metallic mercury directly indicates that photoreduction of mercurous ions to metallic mercury contributes to formation of the photographic image.

On the other hand, lithographic image formation is likely to be due to photooxidation into mercuric ions. Mercuric copoly(vinyl alcoholacrylate) was prepared in the same way as mercurous, by replacing mercurous nitrate with mercuric nitrate. The plates made therefrom did not swell when immersed in a mixed solvent of acetic acid, water, and alcohol. Selective hardening of mercurous copoly(vinyl alcoholacrylate) exposed is thus ascribed to the ionic crosslinking by mercuric ions.

\section{Strength of Ionic Crosslinkages}

As the mercurous ion is generally expressed by $\mathrm{Hg}_{2}{ }^{++}$, there may be crosslinkages of $-\mathrm{COO}^{-} \ldots \mathrm{Hg}^{+} \ldots \mathrm{Hg}^{+} \ldots \mathrm{O}^{-} \mathrm{CO}-$ between polymer chains of mercurous copoly(vinyl alcoholacrylate). After mercurous ions disproportionating on exposure, the crosslinkages will become $-\mathrm{COO}^{-} \ldots \mathrm{Hg}^{++} \ldots \mathrm{O}^{-} \mathrm{CO}-$. A covalent bond is reported to lie between two mercury atoms in the mercurous ion. ${ }^{5}$ If so, the difference of the ionic crosslinking is based on the strength of the ionic bond. In the case of the mercurous cation, two positive charges are distributed on the two mercury atoms, while the mercuric cation has two positive charges on one atom. The electric field induced by the mercurous cation is weaker than that induced by the mercuric cation, so the bond strength between mercurous cation and carboxylate anion is smaller than that between mercuric cation and the anion. Therefore, eq 2 should perhaps be better expressed by the following equation

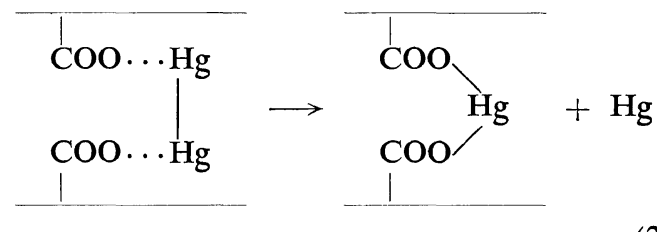

The difference in bond strength may bring about the selective swelling of mercurous copoly(vinyl alcohol-acrylate) unexposed.

The authors wish to thank Professor Rokuro Kuroda of Chiba University for his suggestions on the method of analyzing mercury compounds. Thanks are also due to Professor Takahiro Tsunoda for allowing them use of the monochromator.

\section{REFERENCES}

1. For example, F. Ide and A. Hasegawa, Kobunshi (High Polymers, Japan), 18, 616 (1969); 18, 712 (1969).

2. G. Brauer, "Handbook of Preparative Inorganic 


\section{K. Sugita and S. Suzuki}

Chemistry,"' P. G. Stecher, Translator, Academic Press, London, 1965, p 1120.

3. S. Suzuki and K. Sugita, Bull. Chem. Soc., Japan, 44, 641 (1971).

4. W. R. Sorenson and T. W. Campbell, "Prepara- tive Methods of Polymer Chemistry,' Interscience Publishers, New York, N.Y., 1961, pp 171-173.

5. For example, L. Pauling, "General Chemistry," S. Seki, et al., Translator, Iwanami-shoten, Tokyo, 1958, p 503. 